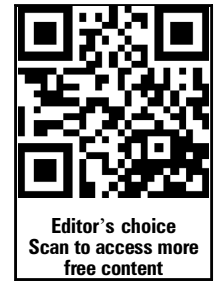

${ }^{1}$ West Virginia University (WVU) School of Medicine, Morgantown, West Virginia, USA

${ }^{2}$ WVU Center for Neuroscience, Morgantown, West Virginia, USA

${ }^{3}$ WVU School of Nursing, Morgantown, West Virginia, USA

${ }^{4}$ West Virginia University Hospitals Department of Neurology, West Virginia University Hospitals, Morgantown, West Virginia, USA

${ }^{5}$ West Virginia University Hospitals Department of Radiology/Interventional Neuroradiology, West Virginia University Hospital,

Morgantown, West Virginia, USA

${ }^{6}$ West Virginia University Prevention Research Center, Morgantown, West Virginia, USA

${ }^{7}$ West Virginia University Department of Emergency Medicine, Morgantown, West Virginia, USA

\section{Correspondence to} Dr T L Barr, WVU Prevention Research Center, One Medical Center Drive, PO Box 9303, Morgantown WV 26506-9303, USA; tlbarr@hsc.wvu.edu

SDB and CS contributed equally to the manuscript.

Received 12 April 2013 Revised 12 September 2013 Accepted 16 September 2013 Published Online First 11 October 2013

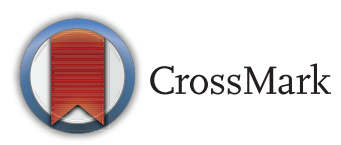

To cite: Brooks SD, Spears $C$, Cummings $C$, et al. J Neurolntervent Surg 2014;6:578-583.

\title{
Admission neutrophil-lymphocyte ratio predicts 90 day outcome after endovascular stroke therapy
}

Steven D Brooks, ${ }^{1,2}$ Chauncey Spears, ${ }^{1,3}$ Christopher Cummings, ${ }^{1,4}$

Reyna L VanGilder, ${ }^{1,3,7}$ Kyle R Stinehart, ${ }^{1}$ Laurie Gutmann, ${ }^{1,4}$ Jennifer Domico, ${ }^{5}$ Stacey Culp, ${ }^{3}$ Jeffrey Carpenter, ${ }^{1,5}$ Ansaar Rai, ${ }^{1,5}$ Taura L Barr ${ }^{1,2,3,6,7}$

\begin{abstract}
Objective Immune dysregulation influences outcome following acute ischemic stroke (AIS). Admission white blood cell (WBC) counts are routinely obtained, making the neutrophil-lymphocyte ratio (NLR) a readily available biomarker of the immune response to stroke. This study sought to identify the relationship between NLR and 90 day AlS outcome.

Methods A retrospective analysis was performed on patients who underwent endovascular therapy for AIS at West Virginia University Hospitals, Morgantown, West Virginia. Admission WBC differentials were analyzed as the NLR. Stroke severity was measured by the National Institutes of Health Stroke Scale (NIHSS) score and outcome by the modified Rankin Scale (mRS) score at 90 days. Univariate relationships between NLR, age, $\mathrm{NIHSS}$, and mRS were established by correlation coefficients; the test was used to compare NLR with recanalization and stroke location (anterior vs posterior). Logistic regression models were developed to identify the ability of NLR to predict mRS when controlling for age, recanalization, and treatment with IV tissue plasminogen activator (TPA).
\end{abstract}

Results 116 patients were reviewed from 2008 to 2011. Mean age of the sample was 67 years, and $54 \%$ were women. Mean baseline NIHSS score was 17 and 90 day mRS score was 4 . There was a significant relationship between NLR and mRS ( $p=0.02)$ that remained when controlling for age, treatment with IV TPA, and recanalization. NLR $\geq 5.9$ predicted poor outcome and death at 90 days.

Conclusions This study shows that the NLR, a readily available biomarker, may be a clinically useful tool for risk stratification when evaluating AlS patients as candidates for endovascular therapies.

\section{INTRODUCTION}

Acute ischemic stroke (AIS) treatment is limited to IV or intra-arterial (IA) tissue plasminogen activator (tPA) and mechanical endovascular therapies. These strategies benefit eligible patients, but carry inherent risks, thereby making determination of individual risk versus benefit important when considering recanalization therapies. The most commonly assessed stroke risk factors are age, infarct volume, and baseline National Institutes of Health Stroke Scale (NIHSS) score. ${ }^{1}{ }^{2}$ Measuring the degree of immune dysfunction immediately following stroke may offer additional prognostic information to help identify which patients will respond most favorably to endovascular intervention.

The neutrophil-lymphocyte ratio (NLR) is an established marker of systemic inflammation, ${ }^{34}$ and has been recently reported as a predictor of 60 day mortality following AIS. ${ }^{5}$ NLR is an indicator of prognosis for cancer, cardiac disease, and sepsis, ${ }^{6-10}$ and has been associated with both the presence and severity of coronary artery disease ${ }^{11}$ and metabolic syndrome. ${ }^{12}$ There is no widely used point of care biomarker proven to predict who will benefit from endovascular therapy, but white blood cell (WBC) counts are routinely obtained during acute stroke triage, making NLR a readily available biomarker.

The objective of this project was to establish a relationship between NLR and stroke outcome in patients who received endovascular therapy and evaluate whether it could serve as a biomarker to predict stroke outcomes. We hypothesized that an elevated NLR would predict poor recovery following stroke. Given the low cost and ease of interpretation, could establish NLR as an acute care biomarker for determining which patients would most benefit from endovascular intervention.

\section{MATERIALS AND METHODS}

\section{Study design and patient selection}

This was a West Virginia University Institutional Review Board approved retrospective analysis of a de-identified database of patients who underwent endovascular therapy for AIS from 2008 to 2011 at West Virginia University Hospitals, Morgantown, West Virginia. WBC differentials were performed on admission as part of the routine clinical workup and were recorded in the patient's medical record. Polymorphonuclear leukocyte (PMN) and lymphocyte counts were analyzed as percentages of the total WBC population. NLR was calculated as the ratio of the percentage of PMNs over the percentage of lymphocytes. Baseline NIHSS score was determined at admission by a staff neurologist. Treatment was classified as having received IV tPA, IA tPA, or mechanical thrombectomy (MT). Outcome was measured by the modified Rankin Scale (mRS) at 90 days during clinical follow-up by trained staff. Additional demographic information was identified from the medical record.

\section{Patient exclusion criteria}

We excluded any patient from the database who developed AIS as a secondary complication to 
another cerebrovascular pathophysiology (aneurysm, hemorrhage; those for which anterior vs posterior location of the stroke was not distinguishable; and patients for whom a baseline WBC differential was not available).

\section{Neuroimaging}

Infarct volume and recanalization defined by the Thrombolysis in Myocardial Infarction (TIMI) grade were determined by a staff interventional neuroradiologist. These data were assessed and graded as previously published. ${ }^{7}$ Recanalization was defined as a TIMI score of 2 or 3 .

\section{Statistical analysis}

Statistical analysis was performed in SPSS (V.20; SPSS Inc, Chicago, Illinois, USA). Descriptive and frequency analyzes were conducted for all demographic and clinical data. Univariate correlations between NLR, age, NIHSS, and the 90 day mRS score were identified by the Pearson correlation coefficient. Relationships between IV tPA and both mRS and NLR were identified by t-test. Relationships between recanalization and both mRS and NLR were also identified by t-test.

\section{Post hoc analysis}

A strong trend between NLR and age was identified; when the data were split into two age groups (patients aged $<80$ vs $\geq 80$ years), the relationship between NLR and age achieved significance in patients $<80$ years. Outcome prediction models and receiver operating characteristic curve (ROC) analysis were then reanalyzed by age group. Data were organized by NLR and mRS, t-test comparisons were conducted, and two significant cut-off points for NLR (3.2 for functional independence and 5.9 for poor outcome) to predict $\mathrm{mRS}$ were identified for patients $<80$ years of age. We defined NLR $\leq 3.2$ as 'low', NLR $>3.2$ but $<5.9$ as 'intermediate', and NLR $\geq 5.9$ as 'high'.

\section{Outcome prediction and ROC analysis}

For prediction analysis, mRS could not be evaluated as an ordinal variable because of sample size restrictions. Therefore, three logistic regression models were developed to identify the ability of NLR $\geq 5.9$ or NLR $\leq 3.2$ to predict 90 day mRS when controlling for age $<80$ years, treatment with IV tPA, and presence of recanalization. In model $1, \mathrm{mRS}$ was dichotomized into an outcome of functional independence (mRS 0-3) versus functional dependence or death (mRS 4-6) to test the ability of NLR $\leq 3.2$ to predict an outcome of functional independence. In model 2 , mRS was again dichotomized as mRS (0-3) versus mRS (4-6) to test the ability of NLR $\geq 5.9$ to predict functional dependence or death. In model $3, \mathrm{mRS}$ was dichotomized as
mRS (0-5) versus mRS (6) to test the ability of NLR $\geq 5.9$ to predict death. The level of significance for these descriptive comparisons was established at 0.05 for two-sided hypothesis testing. ROC and areas under the ROC curves (AUC) were used to test the performance of the logistic regression models.

\section{RESULTS}

A total of 116 patients were reviewed and included in this analysis. Mean age of the patient sample was 68 years (range 18-93) with 54\% being female. Mean baseline NIHSS score was 17 (range 1-48) and median 90 day mRS was 4. Eighty-eight $(75.9 \%)$ patients received IA tPA, $74(63.8 \%)$ patients received MT, and 27 (23.3\%) received IV tPA. One patient did not receive any treatment. Mean PMN percentage was $69.4 \%$ (range $34-89 \%$, SD 10.3\%), and mean lymphocyte percentage was $20.4 \%$ (range $6-60 \%$, SD $8.9 \%$ ) of the total WBC population. Median NLR was 3.74 (range 0.6-17.8). Recanalization was achieved in $54 \%$ of patients (TIMI score 2 or 3). Anterior circulation strokes comprised $82.5 \%$ of the population. Demographic information, with NLR presented as low and mid range (NLR $<5.9$ ) or high (NLR $\geq 5.9$ ), is presented in table 1.

Univariate analysis revealed the following significant correlations: age and outcome $(\mathrm{r}=0.34, \mathrm{p}=0.001)$, NIHSS and outcome $(\mathrm{r}=0.41, \mathrm{p}<0.0001)$, and NLR and outcome $(\mathrm{r}=0.24$, $\mathrm{p}=0.018)$. NLR was also significantly correlated with NIHSS score $(\mathrm{r}=0.38 ; \mathrm{p}<0.0001)$ and there was a trend for a correlation with age $(r=-0.16 ; p=0.09)$. NLR was not significantly different according to treatment type (IA tPA, IV tPA, or MT). Stroke location (anterior vs posterior) was not related to NLR or outcome in our patient population $(\mathrm{p}=0.2)$. Patients who recanalized $(t=4.3 ; \mathrm{p}<0.001)$ or received IV tPA $(t=4.2 ; \mathrm{p}<0.001)$ were more likely to have a good outcome but there was no relationship with NLR for either recanalization or IV tPA.

\section{Cut-off point for NLR}

We identified 28 patients with NLR $\geq 5.9$, of whom 25 had a poor outcomes (90 day mRS 4-6). As such, we established NLR $\geq 5.9$ as a preliminary cut-off point for poor outcome (mRS 4-6) following AIS $\left(\chi^{2}=4.7 ; p=0.024\right)$. The three exceptions are described in the discussion. We also found another cut-off point where patients with NLR values $\leq 3.2$ were more likely to have a functionally independent outcome (mRS 0-3) $\quad\left(\chi^{2}=43.9\right.$; $\mathrm{p}=0.04)$.

\section{NLR and outcome}

In univariate analysis, higher PMN values $(r=0.29, p=0.003)$ and lower lymphocyte values $(\mathrm{r}=-0.32, \mathrm{p}=0.001)$ were

Table 1 Patient demographic information by neutrophil-lymphocyte ratio

\begin{tabular}{|c|c|c|c|c|}
\hline & Total $(n=116)$ & Low $\operatorname{NLR}+(n=88)$ & High NLR $\ddagger(n=28)$ & Statistic/p value \\
\hline Age (years) (mean range) & $67(18-93)$ & $68.9(18-93)$ & $61.9(25-87)$ & $\mathrm{t}=2.01 ; 0.05^{*}$ \\
\hline NIHSS score (mean range) & $17(1-48)$ & $15(1-42)$ & $22(8-48)$ & $\mathrm{t}=-3.6:<0.001^{*}$ \\
\hline mRS score (mean range) & $4(0-6)$ & $3(0-6)$ & $5(0-6)$ & $\mathrm{t}=-2.4 ; 0.016^{*}$ \\
\hline IV tPA (n \%) & $27(23.3)$ & $23(26.1)$ & $4(14.3)$ & $\chi^{2}=1.7 ; 0.19$ \\
\hline +Recanalization (n \%) & $41(54.7)$ & $32(36.4)$ & $9(32.1)$ & $\chi^{2}=1.03 ; 0.31$ \\
\hline Anterior location (n \%) & $94(83.9)$ & $74(84.1)$ & $20(71.4)$ & $\chi^{2}=3.1 ; 0.07$ \\
\hline
\end{tabular}


significantly correlated with death and worse stroke outcome. We generated three predictive models using the NLR cut-off points to test their prognostic value. For model $1, \mathrm{NLR} \leq 3.2$ significantly predicted functional independence (mRS $0-3$ ) $(\mathrm{OR}=9.69, \mathrm{p}=0.013)$ when controlling for age, IV tPA, and recanalization. In ROC analysis, model 1 had an $\mathrm{AUC}=0.87$ (Figure $1 \mathrm{~A}$ ). In model $2, \mathrm{NLR} \geq 5.9$ significantly predicted functional dependence and poor outcome (mRS 4-6) $(\mathrm{OR}=6.72$, $\mathrm{p}=0.025$ ) when controlling for age, IV tPA, and recanalization.
The AUC for model 2 was 0.87 (Figure 1B). In model 3, NLR $\geq 5.9$ significantly predicted death (mRS 6) $(\mathrm{OR}=6.69$, $\mathrm{p}=0.006$ ) when controlling for age, IV tPA, and recanalization. The AUC for model 3 was 0.80 (Figure 1C). Multivariate analysis for each model is summarized in table 2 .

To demonstrate the significance of NLR in predicting functional independence, we identified the ability of NLR as a linear variable to predict mRS. When $\mathrm{mRS}$ was dichotomized into mRS (0-3) and mRS (4-6), NLR as a linear variable remained a
A

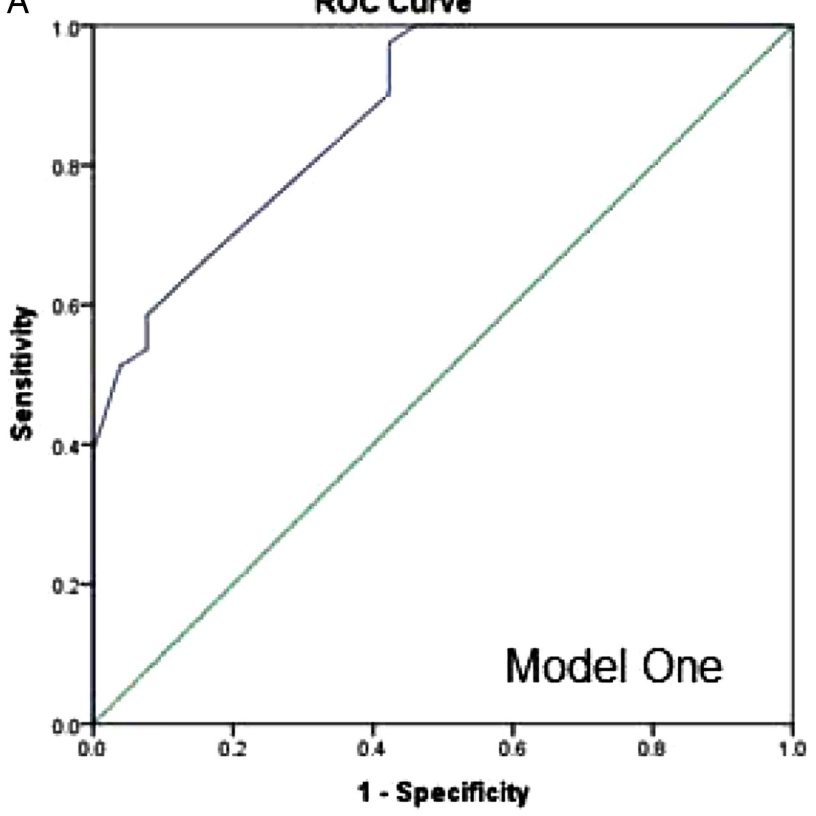

C

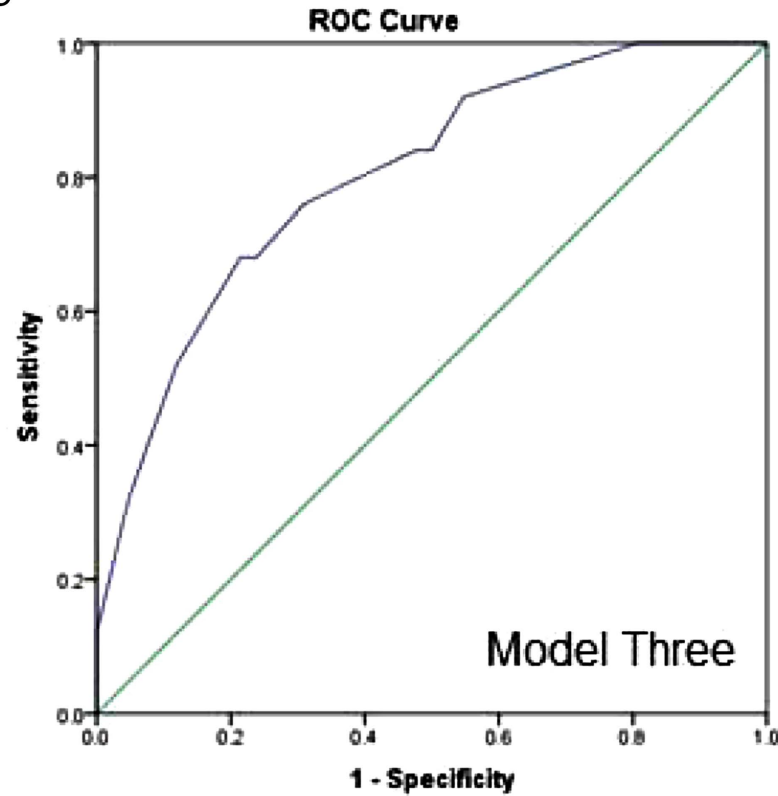

B

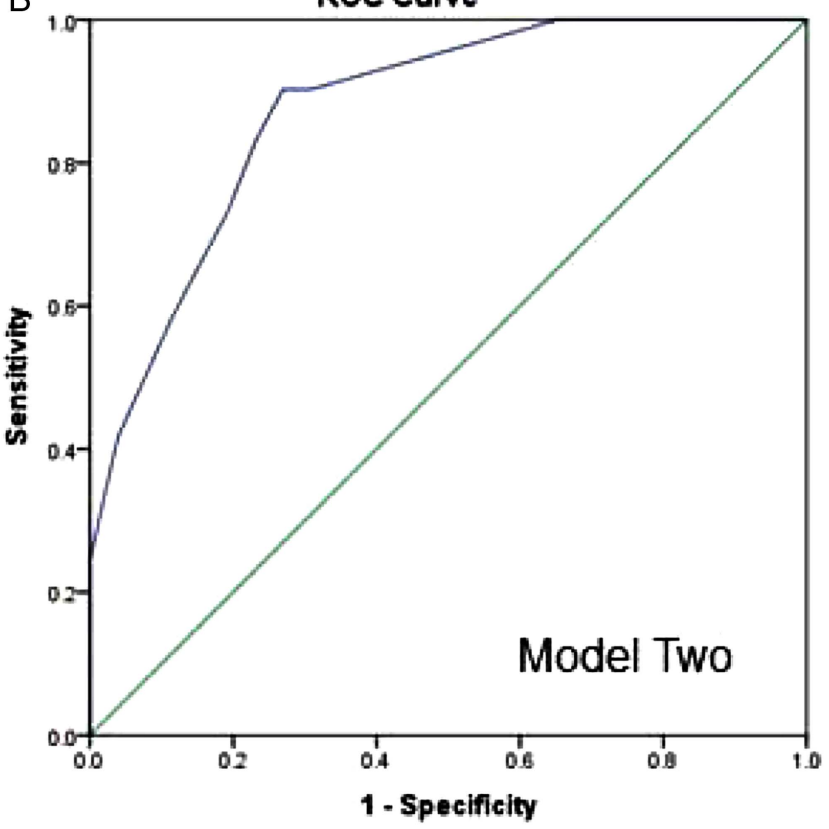

D

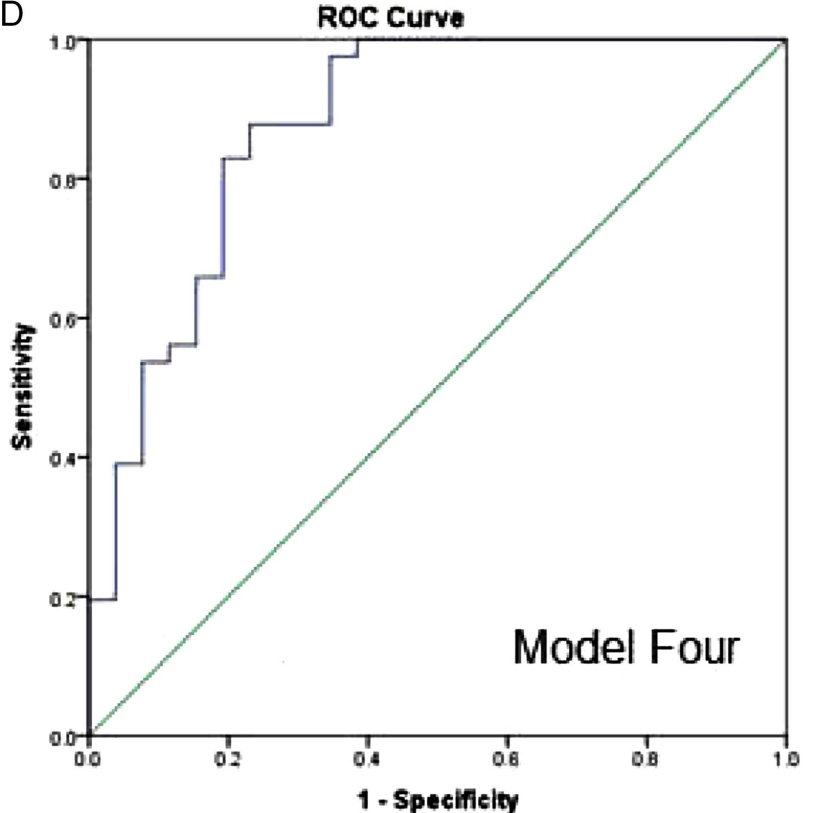

Figure 1 Receiver operating characteristic curve (ROC) analysis for all predictive models in patients $<80$ years. (A) Model 1 evaluated the ability of the neutrophil-lymphocyte ratio (NLR) $\leq 3.2$ to predict a functionally independent outcome (modified Rankin Scale (mRS) score of 0-3) when controlling for age, recanalization, and treatment with IV tissue plasminogen activator (tPA). (B) Model 2 evaluated the ability of NLR $\geq 5.9$ to predict a functionally poor outcome and death (mRS score 4-6) when controlling for age, recanalization, and treatment with IV tPA. (C) Model 3 evaluated the ability of NLR $\geq 5.9$ to predict an outcome of death by dichotomizing patients into mRS $0-5$ or mRS 6 when controlling for age, recanalization, and treatment with IV tPA. (D) Model 4 evaluated the ability of the NLR as a linear variable to predict either a functionally independent outcome or poor outcome by dichotomizing patient 90 day mRS into mRS (0-3) or mRS (4-6) while controlling for age, recanalization, and treatment with IV tPA. 
Table 2 Multivariate analysis for neutrophil-lymphocyte ratio outcome prediction models, adjusted for age, IV tissue plasminogen activator, and recanalization

\begin{tabular}{lcc}
\hline Variable & OR $(95 \% \mathrm{Cl})$ & p Value \\
\hline Results for model 1: mRS (0-3) vs (4-6) & \\
NLR $\leq 3.2$ & $9.69(1.61$ to 58.24$)$ & 0.013 \\
Age $\geq 80$ years & $0.140(0.17$ to 17.13$)$ & 0.067 \\
Recanalization & $20.25(3.54$ to 115.8$)$ & 0.001 \\
IV tPA & $6.48(1.07$ to 39.32$)$ & 0.042 \\
Results for model 2: mRS (0-3) vs (4-6) & \\
NLR $\geq 5.9$ & $6.73(1.27$ to 35.73$)$ & 0.025 \\
Age $\geq 80$ years & $4.09(0.722$ to 23.22$)$ & 0.111 \\
Recanalization & $0.076(0.02$ to 0.345$)$ & 0.001 \\
IV tPA & $0.171(0.032$ to 0.903$)$ & 0.038 \\
Results for model 3: mRS (0-5) vs $(6)$ & \\
NLR $\geq 5.9$ & $6.69(1.7$ to 26.3$)$ & 0.006 \\
Age $\geq 80$ years & $4.25(1.05$ to 17.13$)$ & 0.04 \\
Recanalization & $0.27(0.08$ to 0.89$)$ & 0.03 \\
IV tPA & $0.65(0.12$ to 3.39$)$ & 0.61 \\
\hline mRS, modified Rankin Scale; NLR, neutrophil-lymphocyte ratio; tPA, tissue & \\
plasminogen activator. &
\end{tabular}

significant predictor of 90 day $\mathrm{mRS}(\mathrm{p}=0.03)$ when controlling for age, IV tPA, and recanalization. The AUC for model 4 was 0.88 (Figure 1D).

\section{Interaction between NLR and age}

There was a significant interaction between age and NLR for patients $<80$ years of age. When patients of $\geq 80$ years of age were analyzed separately, none of the variables significantly predicted outcome in any of the models; this included NLR $\geq 5.9$ or $\leq 3.2$, recanalization, and treatment with IV tPA. However, in patients $<80$ years of age, NLR $\geq 5.9$ significantly predicted functional dependence and poor outcomes (model 2, $\mathrm{p}=0.026$; $\mathrm{AUC}=0.87$ ) and death (model 3, $\mathrm{p}=0.018$; $\mathrm{AUC}=0.78$ ) when controlling for recanalization and treatment with IV tPA.

\section{DISCUSSION}

This study suggests that NLR, a readily available and inexpensive test, may be a clinically useful predictor of outcome in AIS patients being considered for endovascular recanalization therapy. Our findings demonstrate that NLR, as a linear variable, can predict functional independence or death. When evaluating by prospective clinical benchmark values, a high NLR $(\geq 5.9)$ predicts poor outcome and death (mRS of 4-6) while a low NLR $(\leq 3.2)$ predicts functional independence (mRS of $0-3$ ), even when controlling for age, treatment with IV tPA, and recanalization. As endovascular interventions carry inherent risks, clinicians must evaluate several parameters, including age, severity, comorbidities, and stroke location to determine eligibility. Our results suggest that NLR significantly predicts outcome and could be used in addition to these variables to identify which patients may benefit from an interventional procedure. NLR may also serve as a tool to help clinicians counsel patients and families when consenting for these procedures.

We chose to define poor outcome as functional dependence and death (mRS of 4-6). In clinical practice, a patient with mRS $(0-3)$ can be considered functionally independent yet many clinical trials define good outcomes as mRS (0-2). This more narrow definition excludes patients who may be functionally independent at 90 days and not universally considered to have a poor outcome; this is especially true for patients of advanced age and patients with pre-existing impairments. However, mRS (4-6) is an accepted definition of a universally poor outcome for all age groups. When utilizing a biomarker that might aid in the decision 'not to treat', it is imperative to be strict in the definition of poor outcome so as not to eliminate patients who may benefit from endovascular therapies. Thus we chose mRS $(0-3)$ as an inclusive measure of functionally independent outcome.

Of note, NLR may also be useful as an inclusion/exclusion criterion when considering patients for enrollment in clinical trials. NLR predicts outcome independently of intervention, including treatment with IV tPA; this would indicate that patients with an elevated NLR may fare poorly during a clinical trial, regardless of treatment, thus adversely impacting the outcome measures of the clinical trial. This is especially important when considering the results of recent clinical trials, such as Interventional Management of Stroke (IMS) III, in which patients were randomized to either IV tPA or IV tPA and endovascular therapy ${ }^{13}$; our results suggest that NLR can predict outcome regardless of IV tPA treatment and could therefore be used as an exclusion criterion when considering patient eligibility for a future trial.

A recent study identified final infarct volume as having a similar predictive ability for outcome. ${ }^{14}$ However, that study evaluated only anterior circulation strokes, and used imaging data up to 2 weeks from onset for outcome prediction. Our study included both anterior and posterior circulation strokes and used only complete blood count differentials taken during admission to predict outcome. NLR is a more readily available predictor of outcome for use in acute clinical decision making, especially in hospitals where acute imaging is not available.

Our data revealed a significant interaction between NLR and age. Age alone is a powerful predictor of outcome and death, making use of predictive biomarkers in older patients more difficult. When analyzing patients by age $<80$ years, we found a very strong relationship between NLR and outcome, but when analyzing by patients $\geq 80$ years of age, NLR, recanalization, and treatment with IV tPA all failed to significantly predict outcome. This may be explained by attenuation of neutrophil function with increased age; decreased rates of neutrophil chemotaxis, phagocytosis, bacterial killing, and cytokine release are all associated with aging. ${ }^{15}$ Additionally, we observed that elderly patients have decreased neutrophil percentage counts compared with younger patients, as well as a general narrowing of the gap between PMN and lymphocyte counts. These observations indicate an overall decreased ability to respond to immune challenges with age, which is of clinical importance when considering treatment options for those patients $\geq 80$ years of age. Interestingly, Yoo et $a l^{14}$ also found that the ability to predict outcome using final infarct volume was much stronger in patients $<80$ years of age, suggesting that predictive biomarkers for stroke may have limited utility in older patients.

A high NLR is reflective of both an elevated innate immune response (higher PMNs) and a decreased adaptive immune response (lower lymphocytes). Similarly, a growing body of evidence supports the presence of systemic immunosuppression following AIS ${ }^{16-20}$; post-stroke infections, including pneumonia and urinary tract infections, ${ }^{16} 1721$ are a major cause of poststroke morbidity and mortality, occurring in up to $65 \%$ of AIS patients. ${ }^{16}$ Stroke induced immune suppression reduces the body's ability to fight antigenic challenges, ${ }^{22}$ and NLR reflects the immune response; we therefore propose that NLR represents immune regulation (or dysregulation) following stroke and 
may predict susceptibility to post-stroke complications. For example, a high NLR could identify patients at increased risk of post-stroke infection, allowing patient care strategies to be adjusted accordingly. ${ }^{23}$ Future studies should measure changes in NLR over time to determine its significance as a biomarker for monitoring response to stroke, including vasogenic edema, apoptosis, hemorrhagic transformation, infectious risk, and other secondary stroke complications.

We identified a cut-off point of NLR $\geq 5.9$ for predicting poor stroke outcome (mRS 4-6). Of the 28 patients with NLR $\geq 5.9,25$ patients had a poor outcome. The three patients with better outcomes were young (mid-20s) (mRS 1), middle aged (around 60 years of age) (mRS 0), and more elderly (mid-70s) (mRS 2). The young patient was a noted smoker of both tobacco and marijuana, and had reported a productive chest cough for 2 weeks prior to stroke. Due to possible pre-stroke infection, and status as a smoker and drug user, it is possible the patient had an elevated baseline NLR prior to his stroke. The middle aged patient suffered from chronic colitis and achieved recanalization after MT. The elderly patient had undergone surgery for tumor resection 1 month prior to the stroke and was still receiving radiation therapy. This patient also recanalized fully after the interventional procedure, which may have influenced the better outcome. As a second potential cut-off point, we observed that in the $<80$ years population, NLR $\leq 3.2$ strongly predicted an outcome of functional independence. Importantly, there were outliers within each of these cut-off point categories, and these numbers are approximate thresholds, as seen within our patient population. Chronic inflammatory conditions, pre-stroke infections, and cancer treatments may all affect a patient's NLR and will need to be taken into account when establishing guidelines for clinical use. Due to our limited study size, a future study with higher power will be needed to establish accurate NLR benchmark values for clinical use.

Tokgoz et $a l^{5}$ recently published a studying showing that NLR $>5$ could predict short term mortality following acute stroke. This study defined short term follow-up as 60 days following stroke, and found that NLR $>5$ predicted mortality within this time frame with a HR of 3.30 (95\% CI 1.35 to 8.07). These results were similar to our findings that NLR $\geq 5.9$ predicted mortality at 90 days with an OR of 6.69 (95\% CI 1.70 to 26.33). We are very encouraged by the overlap in findings, as Tokgoz et al used a larger sample size and did not restrict their study to patients who received endovascular therapy. While both of these studies indicate that baseline NLR can predict clinical mortality for ischemic stroke, our findings expand on this previous study in several ways. First, whereas the previous study reported a correlation between elevated NLR and increased mortality, we have provided a detailed evaluation of the predictive power of NLR using three predictive models: one for predicting good outcome, one for poor outcome, and one for mortality. We also found a significant interaction between advanced age and NLR, which was not included in the previous study. The models presented in this paper provide a more detailed view of how NLR and immune dysregulation affect stroke outcomes.

Our study is limited from broader application due to our selected patient population, as only patients who received endovascular therapy were included in our analysis. Future studies should evaluate NLR as a prognostic biomarker in a larger and more varied cohort of ischemic stroke patients. We also did not collect information on post-stroke complications inducing cerebral edema, hemorrhagic transformation, or infection; these data should be collected in future studies to identify NLR as a biomarker for predicting risk of post-stroke complications. It will be of interest to study the differential immune response in patients $\geq 80$ years of age and factors that may determine response to therapeutics in this population. Finally, it will be important to determine the relationship between cardiovascular conditions, such as metabolic syndrome and atherosclerosis, prestroke infections, NLR, and the physiological link with stroke recovery.

\section{CONCLUSION}

We have shown that NLR, a readily available and inexpensive biomarker often collected in the acute triage assessment of AIS, can predict 90 day outcome in stroke patients who receive endovascular therapy. NLR $\geq 5.9$ predicted poor outcomes and death, while NLR $\leq 3.2$ predicted an outcome of functional independence. Clinicians may use NLR to refine their assessment of risk versus benefit when evaluating patients as candidates for endovascular therapy. NLR may also have utility as an inclusion criterion for future clinical trials. An expanded study of AIS patients is warranted to refine benchmark values for NLR for clinical use, and to validate NLR as a predictor of long term prognosis in patients who do not receive interventional therapies.

Acknowledgements We thank West Virginia University Hospital clinical nursing staff and the Departments of Emergency Medicine, Neurology, Neurosurgery, and Interventional Radiology for their assistance with patient identification and database maintenance. We would like to acknowledge the patients and their families for their time and cooperation.

Contributors SDB contributed to conception and design of the study, acquisition of the data, and analysis and interpretation of the data. SDB was responsible for drafting of the article and revisions. CS contributed to conception and design of the study, acquisition of the data, and analysis of the data. CS contributed to both the drafting and critical revision of the article. CC contributed to conception of the study and analysis of the data. CC contributed to the critical revisions for important intellectual content for this article. RLV contributed to the conception of the study, analysis and interpretation of the data for this study. RLV contributed to the drafting and important revisions of the article. KRS contributed to the acquisition and analysis of the data for this study. KRS contributed to both the drafting and revision of the article. LG contributed to the analysis and interpretation of the data for this study. LG contributed to important intellectual revisions of the article. JD contributed to the design of the study and acquisition of the data. JD contributed to the important intellectual revisions of the article. SC contributed to analysis of the data and was primarily responsible for the statistical analysis for this article. SC contributed to important intellectual revisions of the article. JC contributed to the conception and design of the study, and acquisition and analysis of the data. JC contributed to important intellectual revisions of the manuscript. AR contributed to the conception and design of the study, and acquisition and analysis of the data. AR contributed to important intellectual revisions of the manuscript. TLB contributed to the conception and design of the study, and analysis and interpretation of the data. TLB was responsible for important intellectual revisions to the article. All authors approved the final version of the manuscript for publication.

Funding Supported by WVU Foundation funding and a WV-INBRE grant P20 RR016477 from the NIH National Center for Research Resources supporting the Appalachian Cardiovascular Research Network (to TLB). TLB is a 2012 Robert Wood Johnson Foundation Nurse Faculty Scholar. The project described was supported by the National Institute Of General Medical Sciences, U54GM104942.

Disclaimer The content is solely the responsibility of the authors and does not necessarily represent the official views of the NIH.

Competing interests None.

Ethics approval The study was approved by the institutional review board of West Virginia University.

Provenance and peer review Not commissioned; externally peer reviewed.

Data sharing statement The corresponding author is happy to discuss the de-identified patient database used in this study on request.

\section{REFERENCES}

1 Meyers PM, Schumacher HC, Connolly ES Jr, et al. Current status of endovascular stroke treatment. Circulation 2011;123:2591-601. 
2 Ghobrial GM, Chalouhi N, Rivers L, et al. Multimodal endovascular management of acute ischemic stroke in patients over 75 years old is safe and effective.

J Neurointerv Surg 2013:5(Suppl 1):i33-i37.

3 Walsh SR, Cook EJ, Goulder F, et al. Neutrophil-lymphocyte ratio as a prognostic factor in colorectal cancer. J Surg Oncol 2005:91:181-4.

4 Zahorec R. Ratio of neutrophil to lymphocyte counts — rapid and simple parameter of systemic inflammation and stress in critically ill. Bratisl Lek Listy 2001;102:5-14

5 Tokgoz S, Kayrak M, Akpinar Z, et al. Neutrophil lymphocyte ratio as a predictor of stroke. J Stroke Cerebrovasc Dis Published Online First: 13 Mar 2013. pii: S10523057(13)00017-7. doi:10.1016/j.jstrokecerebrovasdis.2013.01.011

6 Poisson SN, Johnston SC, Josephson SA. Urinary tract infections complicating stroke: mechanisms, consequences, and possible solutions. Stroke 2010;41:e180-4.

7 Prass K, Meisel C, Hoflich C, et al. Stroke-induced immunodeficiency promotes spontaneous bacterial infections and is mediated by sympathetic activation reversal by poststroke T helper cell type 1-like immunostimulation. J Exp Med 2003;198:725-36.

8 Rai AT, Jhadhav Y, Domico J, et al. Procedural predictors of outcome in patients undergoing endovascular therapy for acute ischemic stroke. Cardiovasc Intervent Radiol 2012;35:1332-9.

9 Tamhane UU, Aneja S, Montgomery D, et al. Association between admission neutrophil to lymphocyte ratio and outcomes in patients with acute coronary syndrome. Am J Cardiol. 2008;102:653-7.

10 de Jager CP, van Wijk PT, Mathoera RB, et al. Lymphocytopenia and neutrophillymphocyte count ratio predict bacteremia better than conventional infection markers in an emergency care unit. Crit Care 2010;14:R192.

11 Kaya H, Ertas F, Islamoglu Y, et al. Association between neutrophil to lymphocyte ratio and severity of coronary artery disease. Clin Appl Thromb Hemost Published Online First: 7 Aug 2013. doi:10.1177/1076029613499821
12 Buyukkaya $\mathrm{E}$, Karakas MF, Karakas $\mathrm{E}$, et al. Correlation of neutrophil to lymphocyte ratio with the presence and severity of metabolic syndrome. Clin Appl Thromb Hemost Published Online First: 18 Sept 2013. doi:10.1177/1076029612459675

13 Broderick JP, Palesch YY, Demchuk AM, et al. Endovascular therapy after intravenous t-PA versus t-PA alone for stroke. N Engl J Med 2013:368:893-903.

14 Yoo AJ, Chaudhry ZA, Nogueira RG, et al. Infarct volume is a pivotal biomarker after intra-arterial stroke therapy. Stroke 2012;43:1323-30.

15 Harris NR, Rumbaut RE. Age-related responses of the microcirculation to ischemiareperfusion and inflammation. Pathophysiology 2001;8:1-10.

16 Davenport RJ, Dennis MS, Wellwood I, et al. Complications after acute stroke. Stroke 1996;27:415-20

17 Meisel C, Schwab JM, Prass K, et al. Central nervous system injury-induced immune deficiency syndrome. Nat Rev Neurosci 2005;6:775-86.

18 Elkind MS, Cheng J, Rundek T, et al. Leukocyte count predicts outcome after ischemic stroke: the Northern Manhattan Stroke Study. I Stroke Cerebrovasc Dis 2004:13:220-7.

19 ladecola C, Anrather J. The immunology of stroke: from mechanisms to translation. Nat Med 2011;17:796-808.

20 Jickling GC, Sharp FR. Blood biomarkers of ischemic stroke. Neurotherapeutics 2011;8:349-60.

21 Deguchi T, Takeshita N, Balam TA, et al. Galanin-immunoreactive nerve fibers in the periodontal ligament during experimental tooth movement. J Dent Res 2003:82:677-81.

22 Offner H, Vandenbark AA, Hurn PD. Effect of experimental stroke on peripheral immunity: CNS ischemia induces profound immunosuppression. Neuroscience 2009:158:1098-111.

23 Nardi K, Milia P, Eusebi $\mathrm{P}$, et al. Admission leukocytosis in acute cerebral ischemia: influence on early Outcome. J Stroke Cerebrovasc Dis 2012;21:819-24. 